\title{
3-D Face Modeling from Two Views and Grid Light
}

\author{
Lei Shi, Xin Yang, and Hailang Pan \\ Institute of Image Processing and Pattern Recognition, \\ Shanghai Jiaotong University, Shanghai, 200030, China \\ \{s10030322014, yangxin, panhailang\}@sjtu.edu.cn
}

\begin{abstract}
In this paper, an algorithm for extracting three-dimension shape of human face from two 2D images_using grid light is presented. The grid pattern is illuminated by incandescence light instead of laser in order to protect human eyes or skin and reduce cost. An uncoded grid pattern is projected on human face to solve the problem of correspondence between a pair of stereo images. Two images acquired at same time are smoothed to diminish noise at first. Then grid stripes from these images are extracted and thinned by a marked watershed algorithm. A new method based on graph connectivity to locate and label grid intersections from these images is also presented. According to labeling principles, a set of matched points is build. The set of matched points are further used to calculate three-dimension-depth information of human face. Experiment results show the feasibility of the proposed method.
\end{abstract}

\section{Introduction}

Face modeling is an important problem in many multimedia applications, such as teleconferencing, virtual reality, animation and face recognition. There are several major approaches for face modeling.

For instance, DeCarlo et al.[1] used the anthropometric methods to build a facial model; At first, a manually-constructed B-spline surfaces is constructed, and then surface fitting and constraint optimization is applied to the surface.

In [2], facial model is directly acquired from 3D laser scanners or structured light range sensors. Water's face model is a well-known model by this kind of equipments. In many face modeling methods, the facial model is regarded as a generic model. Kawai et al.[3] presented a method of range data integration based on region segmentation and extraction of feature parameters.

Also facial model is reconstructed by digital equipment, such as low-cost and passive input devices (video cameras or digital camera). For instance, Chen and Medioni [4] built facial model from a pair of stereo images. However, currently it is still difficult to extract sufficient information about the facial geometry only from 2D images. This is the reason why Guenter et al. [5] to utilize a large number of fiducial points to acquire 3D facial geometry.

Though we can acquire 3D information from expensive 3D laser scanners or structured light range sensors, it still takes too much time to scan and detected person must remain stable during the scanning. In order to overcome these problems, recently, some researchers try to incorporate some prior knowledge of facial geometry 
or to make use of a generic facial model. For instance, Ansari, A.-N. et al. [6] deformed a $3 \mathrm{D}$ generic model from two orthogonal views (frontal and profile views) to acquire facial model. Zhang [7] deformed a generic mesh model to an individual's face.

In case a generic facial model can't be provided, some methods integrating structure light and computer vision is applied to human face modeling, such as Andrew Naftel et al. [8] and Philippe Lavoie et al. [9].

Philippe Lavoie et al. [9] proposed a new method for reconstructing 3D facial model from the left and right two-dimensional (2-D) images of an object using a grid of pseudorandom encoded structured light. The proposed method provided three distinctive advantages over a conventional stereo system:1) without new textures;2) less computational intensive;3) solving correspondence problem easily. We draw many inspirations from Philippe Lavoie's method.

Based on an uncoded grid light, our method allows for the introduction of a new procedure for the grid extraction and grid intersection location. The procedure can determine a set of points on the object surfaces on the left and right images with satisfied precision.

In the following, we will describe the main algorithms of this procedure. Section 2 deals with the design and extraction of the projected grid pattern. Section 3 is a description of the extraction process of grid information. $3 \mathrm{D}$ reconstruction of corresponding points from the right and left image is described in Section 4, and experimental results from different angles show the feasibility of our method. Eventually, in Section 5, a conclusion section reviews the main steps and the unique features of this system.

\section{Uncoded Grid Light}

In our method, using a projected grid pattern can capture the whole view of the face, instead of a line or a dot pattern in laser systems that require scanning to ensure covering the whole pattern. Then, a simple scheme is developed which fixes grid stripes along a pair of cross axes. The cross axes is easily distinguished from other stripes in the grid pattern. The uncoded grid light is shown in Fig. 1.

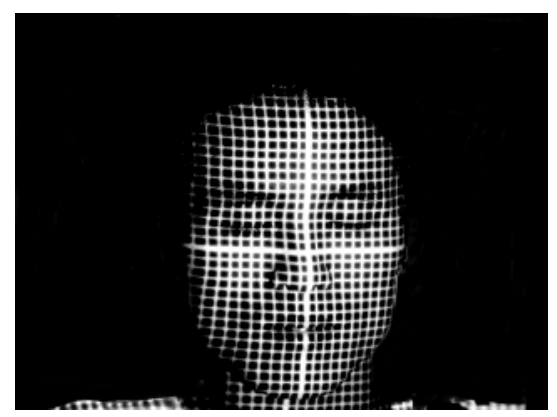

Fig. 1. Uncoded grid light 
The process of extracting interest points is implemented in two steps: extracting the grid and extracting the intersections.

The extraction of grid stripe from the original image is also fulfilled in two steps: smoothing and extracting. The smoothing process consists of applying 2D Gaussian filter with standard deviation of $\sigma=6.5$ (and kernel size $3 \times 3$ ). The filtering is used to eliminate noise signal in original image.

\subsection{Watershed Algorithm}

For most classical 3-Dmodeling methods [9-12,24-28], the projected grid stripes is illuminated by laser because laser is by far the most perfect light source for 3D modeling. The grid stripes can be easily extracted according to brightness, texture or color. The extracted stripes will be thinned by shrinking each stripe to its skeleton in[10,11] or thinning on binary images[12,13,27,28]. In [14], the process is also performed by a least square method approximation of the center points of the lines. Moreover, in [24-26], extracting line edge and line fitting is used to deal with the problem.

But the quality of our grid stripes is worse than classical structure light system because the grid light is illuminated by incandescence instead of laser in our system. The classical methods of extracting and thinning can't meet our requirements. In [9], Philippe Lavoie et al. applied directly the watershed algorithm to extracting and thinning grid stripe because grid stripe has illuminated by laser in this case.

Considering these conditions, our grid extraction process starts with the application of a watershed algorithm [15-17]. The watershed algorithm is applied on the gradient magnitude image. A fast immersion based on the algorithm developed by Vincent and Soille[15] is employed. All pixels in the gradient image are sorted by increasing graylevel values. Once the image is completely flooded, the watershed lines will be obtained. The lines from the watershed algorithm have exactly one pixel wide. Thus, the thinning process is also performed at the same time.

The watershed algorithm can find contiguous edges in an image accurately but suffers from the over-segmentation problem. Consequently, we may obtain many extra details that don't need when grid stripe is segmented, as shown in Fig 2(a). The original image is shown in Fig. 1. There are many methods to deal with oversegmentation, such as prior information [18], classifier [19] and marker [20-22] and so on. But in this paper, selected markers are employed to overcome the problem.

\subsection{Marker Watershed Algorithm}

The marker-based watershed algorithm is a very efficient means for image segmentation and has been used in recent years. In this algorithm flooding starts directly from the marker instead of minima basins. There are some methods to choose right marker. For instance, in [20], S.Beucher et al. applied the marker-controlled watershed to road segmentation and obstacle recognition. These markers have been introduced by hand. Meyer's watershed algorithm [21] floods from two markers so that the final watershed line is located at the highest-crest line only. The result is a more meaningful or a more visually sensible segmentation. Hai Gao et al.[22] extracted marker based on luminance and color information. 
Considering these methods and aiming at our images, a marked watershed algorithm is applied in this paper. As shown in Fig.1, the gray intensity of grid stripes is larger than other parts of original images. In order to remove those unuseful details, we flood directly from edge of grid stripe instead of the original image. The marker image can be expressed as followed:

$$
\left\{\begin{array}{l}
I(x, y) \in M, \text { if }(I(x, y)<\text { Threshold }) \\
I(x, y) \notin M, \text { otherwise }
\end{array}\right.
$$

Where $I(x, y)$ is gray-level intensity of the original image, and $x, y$ is pixel coordinate; $M$ is the set of markers; Threshold is gray-level threshold of pixels in original images. In order to save time and remove unuseful details, the threshold should be selected to approximate the minimum intensity of grid stripes in original image. It can be acquired by Ostu's method [23].

Beginning from the marker image flooding is performed. The right watershed lines will be obtained. The original image is shown in Fig. 1. The extracted grid is shown in Fig. 2 (b) when selected markers are applied.

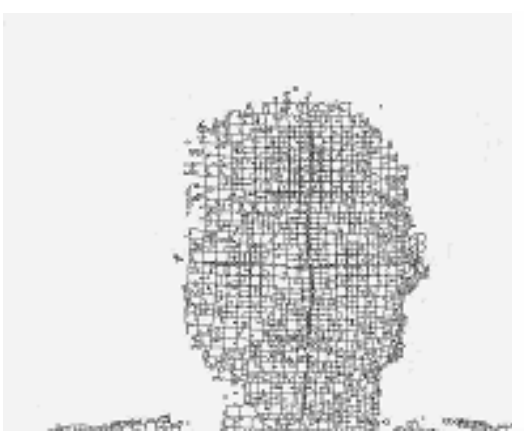

(a)

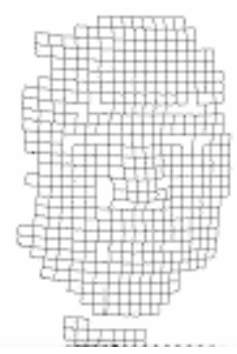

(b)

Fig. 2. Extracted grid

\section{Extracting Grid Information}

More grid information, such as the precise location of these intersection points, their connectivity and their label, are needed after the grid has been exacted by the marker watershed algorithm. To obtain all information, some steps are required.

\subsection{Intersection Detection and Location}

For most classical methods of grid location [9-14,24-28], in general, suitable projector's angle is selected in order to deal with the problem when we have to extract intersections, such as $[25,26]$. In some cases, a conditional set that includes all cases on the extracted grid is proposed. Only those intersections that satisfied one case can 
be regarded as candidates. In those cases, a template whose size is 3 by $3[9,12-14]$ or 7 by $7[10]$ is applied. Pixels with special location can be regarded as candidates in the template.

We have presented a new algorithm based on graph connectivity. Define candidate $P t$ be those pixels lying on the extracted grid stripes (Fig.3). Based on a set of conditions on the set of nonzero pixels in a 3 by 3 square neighborhood centered about the candidate points, these intersections are detected. We call these nonzero pixels the border point $P o$, as shown in Fig.3.

Firstly, our algorithm requires that the set $\mathrm{X}$ of candidates consists of three or four connected border points $P o$ in the square neighborhood, shown in Fig.3(a) and (b).

If two intersection points $P t$ are connected, they are treated as one class. Then the average coordinates $\overline{P t}$ of these intersections $P t i(i=1 \ldots \mathrm{n}$, where $\mathrm{n}$ is the number of intersections from the same class) and the Euclidean distances between the average point $\overline{P t}$ and all intersections $P t i(i=1 \ldots \mathrm{n})$ of a class are calculated. The intersection $P t j$ with minimum Euclidean distance in the class Pti $(i=1 \ldots \mathrm{n})$ will be regarded as the candidate $P t$.

For flat areas, there is one intersection for each node of grid stripes. But for curved areas, there may be more than one intersection from the same node (shown in Fig.3(c)). Secondly, we will calculate $P 3((x 1+x 2) / 2,(y 1+y 2) / 2))$ if two intersections $P 1(x 1, y 1), P 2(x 2, y 2)$ from the same node are so close to each other that the distance between them is no more than 5 pixels, then will replace these intersections by new candidate $P 3$.

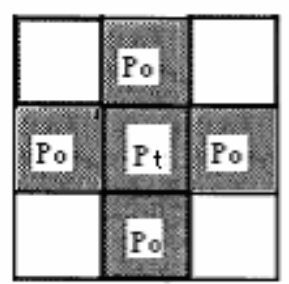

(a)

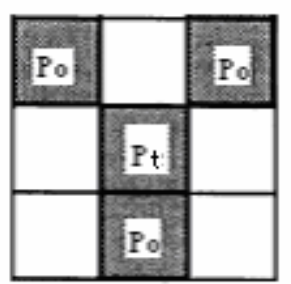

(b)

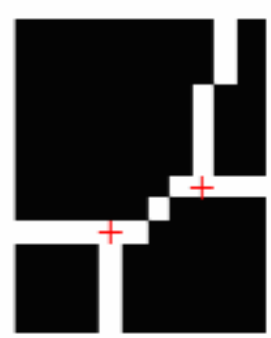

(c)

Fig. 3

\subsection{Intersection Labeling}

A key step in the 3-D reconstruction using grid light is to label these intersections in each 2D image (the stereo correspondence problem). For classical methods [9-14,2428], coded grid pattern is used to deal with 3-D reconstruction problem according to pattern [9-12,24,28] or color [13,14,24-27]. This leads higher cost. We solve the problem by labeling all grid intersections before matching the left and right images. In [9], a labeling methods based on line can be found out. Labeling methods based on point is applied in [10]. We present a labeling method integrating line and point labeling by three steps: 
1) Locating the cross axes;

2) Finding out all grid intersections on the cross axes, and then labeling these grid intersections;

3) Labeling all grid intersections based on labeled grid intersections.

The first step is to locate the cross axes in the original image. We can locate very easily the cross axes because its gray intensity and stripe width is more than that of other stripes. The erosion and dilation are employed in our method.

The second step is to find out intersections $P t$ on the cross axes. By comparing the sets $X$ of all grid intersections with the locations of cross axes, we can find out that the set $X c$ of all grid intersections on the cross axes. The intersection of center Pt on the cross axes will be regarded as the principle point in the set $X c$, and other intersections on the cross axes will be labeled referenced on the principle point $P c$.

The final step is to label all grid intersections based on these labels of labeled grid set $X c$ on the cross axes. Since we have labeled the grid intersections on the cross axes, labeling set of grid intersections $X$ can be obtained by using the method in [9].

\section{Calculating 3D Information}

In most classical structure light system, 3-D information is acquired as a result of a triangulation procedure. In order to avoid the risk of occlusion, binocular vision is applied to acquire 3D information of the object in our system.

Original left image and right image are acquired from two digital cameras. The resolution of cameras is $1024 \times 768$ in our system. The two cameras' intrinsic parameters and the relative position between the two cameras can be acquired by calibration. The results from binocular vision will be used to show the feasibility of our system. Original right and left image are shown in Fig. 4.

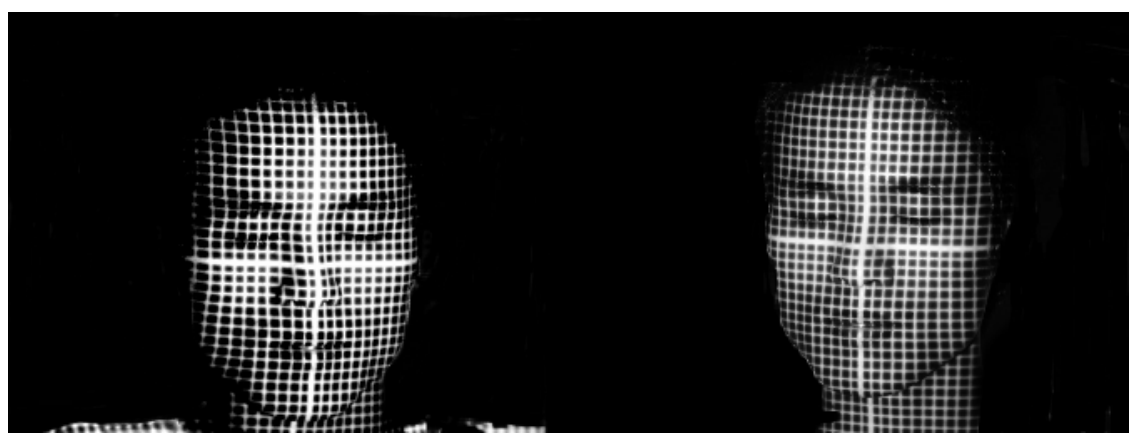

(a) left image

(b) right image

Fig. 4. Original images

3D face information is calculated by binocular vision. When two images of human face are taken, a 3D information map can be easily obtained. The disparity of a point gives a scaled version of its $3 \mathrm{D}$ information. The result from authors' system is shown in Fig.5. 


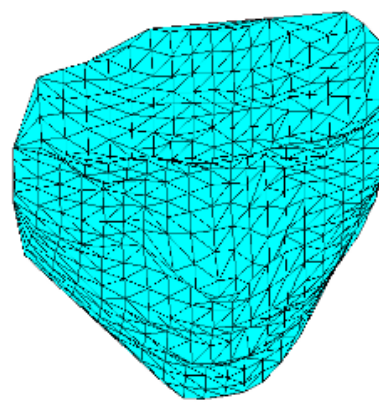

(a)

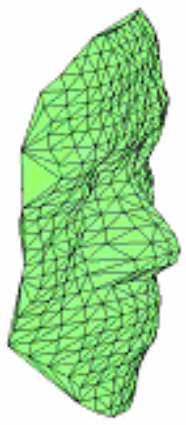

(b)

Fig. 5. The final 3D reconstruction results

\section{Conclusion}

In this paper, an algorithm to recover 3-D information of human face from two 2-D images of the same object is suggested by using incandescence light system consisting of a projector and two digital cameras. The grid pattern in our system facilitates the matching of the similar points situated on the two 2-D images. The resulted matched points help to determine the depth map.

\section{References}

1. D. DeCarlo, D. Metaxas, and M. Stone, An anthropometric face model using variational techniques. In Proc. SICGRAPH, pages 67-74, July 1998

2. F.I. Parke and K. Waters, Appendix 1: Three-dimensional muscle model facial animation. Computer Facial Animation, September 1996

3. Y. Kawai, T. Ueshiba, T. Yoshimi, and M. Oshima, Reconstruction of 3D Objects by Integration of Multiple Range Data. In Proc.11th International Conference Pattern Recognition, I: 154-157, 1992

4. Q. Chen and G. Medioni, Building human face models from two images. In Proc.IEEE 2nd Workshop Multimedia Signal Processing, pages 117-122, December 1998.

5. B. Guenter, C. Grimm, D. Wood, H. Malvar, and F. Pighin, Making faces. In Proc. SIGGRAPH, pages 55-66, July 1998.

6. A-Nasser Ansari, 3D face modeling using two orthogonal views and a generic model. In Proc. International Conference on Multimedia and Expo, 3:289-92, July 6-9, 2003

7. Z. Zhang, Image-based modeling of objects and human faces. In Proc. SPIE, Volume 4309, January 2001

8. A. Naftel and Z. Mao, Acquiring Dense 3D Facial Models Using Structured-Light Assisted Stereo Correspondence, Technique Report, Department of Computation, UMIST, 2002

9. P. Lavoie, D. Ionescu, and E. M. Petriu, 3-D Object Model Recovery From 2-D Images Using Structured Light. IEEE Transactions on Instrumentation and Measurement, 53(2):437-443, April 2004

10. Stanley M. Dunn, Richard L. Keizer and Jongdaw Yu, Measuring the Area and Volume of the Human Body with Structured Light, IEEE Transactions on Systems, Man And Cybernetics, 19(6): 1350-1354, Nov/Dec 1989, 
11. Petriu, E.M.; Sakr, Z.; Spoelder, H.J.W.; Moica, A., Object recognition using pseudorandom color encoded structured light, In Proc. the 17th IEEE Instrumentation and Measurement Technology Conference, 3:1237 - 1241, 1-4 May 2000

12. Guisser, L. Payrissat, R. Castan, S, A new 3-D surface measurement system using a structured light, In Proc. IEEE Computer Society Conference on Computer Vision and Pattern Recognition, pages 784-786, 15-18 Jun 1992

13. G. Hu , G. Stockman, 3-D Surface Solution Using Structured Light and Constraint Propagation, IEEE Transactions on Pattern Analysis and Machine Intelligence, 11(4):390402, April 1989

14. P. Lavoie, D. Ionescu, and E. M. Petriu, A high precision 3D object reconstruction method using a color coded grid and nurbs, In Proc. the International Conference on Image Analysis and Processing, pages 370--375, Venice, Italy, September 1999

15. V. Luc and P. Soille, Watersheds in Digital Spaces: An Efficient Algorithm Based on Immersion Simulations, IEEE Transactions on Pattern Analysis and Machine Intelligence, 13(6):583-598, 1991

16. Paul R. Hill, C. Nishan Canagarajah and David R. Bull, Image Segmentation Using a Texture Gradient Based Watershed Transform, IEEE Transactions on Image Process, 12(12):1618-1634, 2003

17. Roerdink and Meijster, The Watershed Transform: Definitions, Algorithms and Parallelization Strategies, FUNDINF: Fundamental Information, 41:187-228, 2001

18. Susan Wegner, Klaus-Peter Pleissner, Helmut Oswald and Eckart Fleck, Hierarchical watershed transformation based on a-priori information for spot detection in 2D gel electrophoresis images, In Proc. SPIE Int. Soc. Opt. Eng. 3661:749,1999

19. Thierry Géraud, Pierre-Yves Strub, and Jérôme Darbon, Color Image Segmentation Based on Automatic Morphological Clustering, In Proc. IEEE International Conference on Image Processing (ICIP'2001), 3:70-73, Thessaloniki, Greece, October 2001

20. S.Beucher and M. Bilodeau, Road segmentation and obstacle recognition by a fast watershed transformation, Intelligent Vehicles Symposium'94, pages 296-301, 0ctober 1994

21. F. Meyer, Color Image Segmentation, 4th IEEE Conference on Image Processing and Applications, 354:53, pages 303-306 (1992)

22. Hai Gao; Wan-Chi Siu; Chao-Huan Hou, Improved techniques for automatic image segmentation, IEEE Transactions on Circuits and Systems for Video Technology, 11(12):1273 - 1280, Dec. 2001

23. N. Otsu, A Threshold Selection Method from Gray-Level Histograms, IEEE Transactions on Systems, Man, and Cybernetics, 9(1): 62-66, 1979

24. D. Bergmann, New approach for automatic surface reconstruction with coded light, In Proc. Remote Sensing and Reconstruction for ThreeDimensional Objects and Scenes, 2572: 2--9. SPIE, August 1995.

25. G. Stockman and G. Hu, Sensing 3-D surface patches using a projected grid, Computer. Vision Pattern Recognition, pages 602-607, 1986

26. N. Shrikhande and G. Stockman, Surface orientation from a projected grid, IEEE Trans. Pattern Anal. Mach. Intel. , 11(6):650-655, 1989

27. J. Tajima and M. Iwakawa, 3-D data acquisition by rainbow range finder, In Proc. International Conference on Pattern Recognition, pages 309-313, 1990

28. H. S . Yang, K. L. Boyer, and A. C. Kak, Range data extraction and interpretation by structured light, Purdue Univ, West Lafayette, In Tech. Rep., pages 199-205, 1984. 